\title{
THE
}

\section{Taming the Rugged Landscape: Production, Reordering, and Stabilization of Selected Cluster Inherent Structures in the $\mathrm{X}_{13-n} \mathrm{Y}_{\mathrm{n}}$ System}

Dubravko Sabo

J. D. Doll

David L. Freeman

University of Rhode Island, dfreeman@uri.edu

Follow this and additional works at: https://digitalcommons.uri.edu/chm_facpubs

Terms of Use

All rights reserved under copyright.

\section{Citation/Publisher Attribution}

Sabo, D., Doll, J. D., \& Freeman, D. L. (2004). Taming the Rugged Landscape: Production, Reordering and Stabilization of Selected Cluster Inherent Structures in the X13-nTn System. Journal of Chemical Physics, 121(2):847-855. doi: 10.1063/1.1759619

Available at: http://dx.doi.org/10.1063/1.1759619

This Article is brought to you for free and open access by the Chemistry at DigitalCommons@URI. It has been accepted for inclusion in Chemistry Faculty Publications by an authorized administrator of DigitalCommons@URI. For more information, please contact digitalcommons-group@uri.edu. 


\title{
Taming the rugged landscape: Production, reordering, and stabilization of selected cluster inherent structures in the $X_{13-n} Y_{n}$ system
}

\author{
Dubravko Sabo a) and J. D. Doll \\ Department of Chemistry, Brown University, Providence, Rhode Island 02912 \\ David L. Freeman \\ Department of Chemistry, University of Rhode Island, Kingston, Rhode Island 02881
}

(Received 22 March 2004; accepted 19 April 2004)

\begin{abstract}
We present studies of the potential energy landscape of selected binary Lennard-Jones 13 atom clusters. The effect of adding selected impurity atoms to a homogeneous cluster is explored. We analyze the energy landscapes of the studied systems using disconnectivity graphs. The required inherent structures and transition states for the construction of disconnectivity graphs are found by combination of conjugate gradient and eigenvector-following methods. We show that it is possible to controllably induce new structures as well as reorder and stabilize existing structures that are characteristic of higher-lying minima. Moreover, it is shown that the selected structures can have experimentally relevant lifetimes. (C) 2004 American Institute of Physics.
\end{abstract}

[DOI: 10.1063/1.1759619]

\section{INTRODUCTION}

The science of chemistry is characterized by an interplay of reductionist and constructionist themes. On the one hand, one seeks to reduce complex systems to more understandable and more controllable components. On the other, one strives to utilize these components to construct complex assemblies to meet specific chemical, biological, and/or materials goals. The core elements of such efforts have, in the past, been largely atomic and/or molecular in nature.

Viewed from the above perspective, a central component of much ongoing research has been the development of a variety of "reductionist" classical and quantum-mechanical tools. Common goals in the application of these tools have been locating the minimum energy configuration for the associated potential energy surface ${ }^{1}$ and sampling the relevant, low-lying local minima. ${ }^{2-5}$

Increasingly, both chemistry and materials science are entering an era in which the fundamental components of the constructionist phase of the problem often are themselves complex, preassembled objects. Sun et al., ${ }^{6}$ for example, have shown that novel magnetic materials can be prepared through the self-assembly of colloidal clusters. In related developments Lehmann and Scoles ${ }^{7}$ as well as Miller et al. ${ }^{8-10}$ have demonstrated the effectiveness of superfluid solvents in preparing unusual, metastable species. Sustained progress in this emerging field of cluster assembled materials ultimately rests on the ability to characterize and control a broad range of increasingly complex nanoscale objects.

In a previous paper, ${ }^{11}$ we have examined a number of theoretical issues of general concern with respect to predicting/characterizing/controlling the structure and dynamics of cluster-based precursors. That work, in essence,

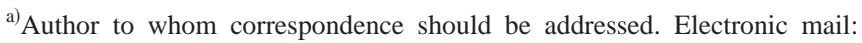
dubravko_sabo@brown.edu
}

seeks to invert the logic of the minimization problem. That is, instead of searching for the minimum energy structures of specified energy landscapes, we strive instead to reshape those landscapes and thereby to exercise control over selected physical systems. In particular, we seek to stabilize and/or kinetically trap conformers of the parent homogeneous system that are otherwise either unstable or metastable. Our hope is that such new structures might have interesting physical properties (electronic, magnetic, optical, thermal, etc.) and can be used as precursors in subsequent assembly procedures. Our previous work ${ }^{11}$ has demonstrated that for small Lennard-Jones systems we could, using selected impurities, both alter the energy ordering of the stable core atom isomers and induce wholly new conformers not seen in the original homogeneous species. We have also seen similar reordering effects in our studies of molecular nitrogen adsorbates on nickel clusters. ${ }^{12}$

In the present study, we wish to extend the results of our previous investigation: First, we wish to consider a number of larger clusters to demonstrate the general applicability of our efforts. Second, we wish to demonstrate the resulting species can be made sufficiently robust that they are of practical interest. To attain the second goal we enhance our previous publication ${ }^{11}$ by determining Rice-Ramsperger-KasselMarcus (RRKM) isomerization rate constants and lifetimes for the generated isomers.

An important byproduct of the current work is the information we garner concerning the structure of the potential energy surfaces of the mixed cluster systems. The mixed clusters $^{13-16}$ show a much richer behavior than their homogeneous counterparts. The energy landscape of the mixed clusters displays a drastic increase in complexity which is manifested by numerous new low-lying minima, i.e., by the abundance of the new structural forms. In the current work we find that many of the disconnectivity graphs have doublefunnel structures. The thermodynamic properties of other 
cluster systems with double-funnel structures ${ }^{2,17}$ can be rich exhibiting solidlike to solidlike phase change phenomena. Motivated by these past studies and the disconnectivity graphs investigated in the current work, in a companion paper $^{18}$ we investigate the energy and heat capacity of some of the systems studied here.

The remainder of the paper is organized as follows: An outline of the computational details of this work is presented in Sec. II. We discuss the methods utilized to find the inherent structures and transition states on the potential energy surface as well as the method to estimate the inherent structures lifetimes. In Sec. III we present the results that demonstrate "proof of principle" with respect to the goals of the present studies. In Sec. IV we summarize our results and speculate about likely future research directions.

\section{COMPUTATIONAL DETAILS}

In this section, we describe the computational details of the studies involving binary clusters of the form $X_{13-n} Y_{n}$. Our overall interest is to explore the extent to which we can utilize the "adatoms" (i.e., the $Y$ system) to induce, reorder, and stabilize selected inherent structures in the "core" $X$ system. While one can easily imagine applications involving more and more complex components, we have found that these relatively simple, two-component clusters are a convenient starting point for an initial study of the issues we raise. $^{11}$

The total potential energy, $V_{\text {tot }}$, of a cluster consisting of $N$ particles is modeled as a pairwise sum of Lennard-Jones interactions

$$
V_{\mathrm{tot}}=\sum_{i<j}^{N} v_{i j}\left(r_{i j}\right)
$$

where the pair interaction as a function of the distance between particles $i$ and $j, r_{i j}$, is given by

$$
v_{i j}\left(r_{i j}\right)=4 \epsilon_{i j}\left[\left(\frac{\sigma_{i j}}{r_{i j}}\right)^{12}-\left(\frac{\sigma_{i j}}{r_{i j}}\right)^{6}\right] .
$$

In Eq. (2) the constants $\epsilon_{i j}$ and $\sigma_{i j}$ are the energy and lengthscale parameters for the interaction of particles $i$ and $j$.

For a binary system, both the "like" $(X-X, Y-Y)$ as well as the "unlike" $(X-Y)$ interactions have to be specified. With an eye toward studying trends in the results as opposed to results for particular physical systems, it is convenient to reduce the number of free parameters. To do so, we shall assume in the present study that the unlike Lennard-Jones values are obtained from the like Lennard-Jones parameters via usual combination rules ${ }^{19}$

$$
\begin{aligned}
& \sigma_{X Y}=\frac{1}{2}\left(\sigma_{X X}+\sigma_{Y Y}\right), \\
& \epsilon_{X Y}=\sqrt{\epsilon_{X X} \epsilon_{Y Y}} .
\end{aligned}
$$

Furthermore, we note that with the mixed Lennard-Jones parameters specified as in Eqs. (3) and (4), the inherent structure topography of the "reduced" potential energy surface of the binary system (i.e., $V_{\text {tot }} / \epsilon_{X X}$ ) is a function of only two parameters $(\sigma, \epsilon)$, the ratios of the corresponding adatom/core length and energy parameters

$$
\begin{aligned}
& \sigma=\sigma_{Y Y} / \sigma_{X X}, \\
& \epsilon=\epsilon_{Y Y} / \epsilon_{X X} .
\end{aligned}
$$

If necessary for a discussion of a specific physical system, the absolute bond lengths, energies, activation energies, etc., can be obtained from the corresponding reduced results by a simple rescaling with the appropriate core-system Lennard-Jones parameters.

\section{A. Stationary points and the disconnectivity graph}

The computational task in our study is thus one of exploring and characterizing the (reduced) potential energy surface of our binary cluster systems as a function of the number of (core, adatom) particles $(n, m)$, and for given $(\sigma, \epsilon)$ ratios. In typical applications the lowest $N_{\text {IS }}$ inherent structures and the associated disconnectivity graphs are determined. For the applications reported here, $N_{\text {IS }}$ is generally of the order of several thousands or more. The inherent structures are found either via conjugate gradient methods ${ }^{20}$ starting from randomly chosen initial configurations, or by more systematic surface exploration methods. ${ }^{21,22}$ In all cases, the inherent structures that are located are confirmed to be stable minima via a standard Hessian analysis. To reduce the chance we miss particular local or global minima, we monitor the number of times individual inherent structures are found and demand that each of the $N_{\text {IS }}$ inherent structures be located a minimum number of times (at least ten) before we terminate our search. Once we are satisfied we have located the relevant inherent structures, transitions states linking these stable minima are obtained using the eigenvector following methods outlined by Cerjan and Miller ${ }^{23}$ and further developed by Simons et al., ${ }^{24-26}$ Jordan $_{\text {et }}$ al. ${ }^{22}$ and Wales. ${ }^{27}$ Finally, with the given inherent structures and transition states, we perform a disconnectivity analysis. ${ }^{28,29}$

\section{B. Rate constants and lifetimes of the inherent structures}

From the known inherent structures and the transition states that connect/separate them, we estimate rates for transitions between neighboring inherent structures. The rate constants allow us to calculate the average amount of time the system will spend in a given inherent structure, i.e., the lifetime of an inherent structure.

There are variety of methods available to estimate the rate constants (see Ref. 30 and references therein). We utilize the harmonic approximation to the RRKM method. It has been found that this method gives good estimates of rates for isomerization of clusters. ${ }^{31}$ The rate constant, $k_{i j}$, for transition leading from inherent structure $j$ to inherent structure $i$ is given as a sum over all transition states connecting inherent structures $i$ and $j$ (Refs. 1 and 32)

$$
k_{i j}=\sum_{\alpha} k_{j}^{\alpha},
$$

where $k_{j}^{\alpha}$ is given by

$$
k_{j}^{\alpha}=\frac{h_{j}^{\alpha} \Pi_{l=1}^{3 N-6} \nu_{l, j}^{\mathrm{IS}}}{h_{j} \Pi_{l=1}^{3 N-7} \nu_{l, j}^{\mathrm{TS}, \alpha}} e^{-\Delta \phi_{j} / k_{B} T},
$$




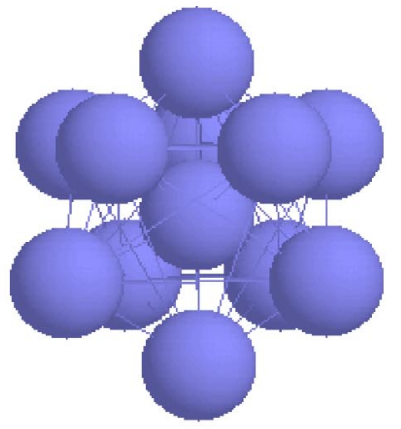

(a)

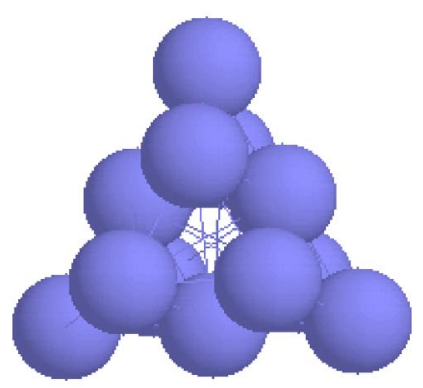

(c)

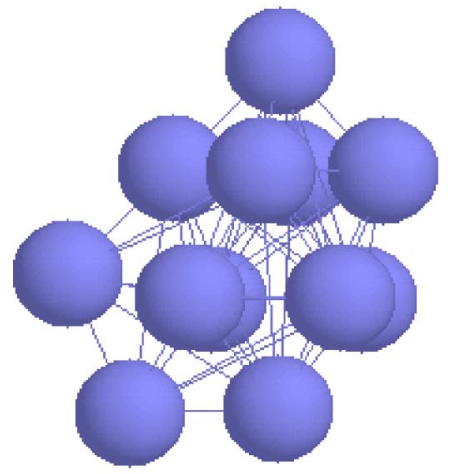

(e)

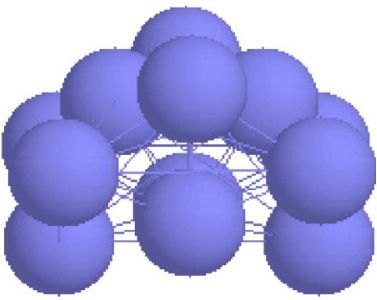

(b)

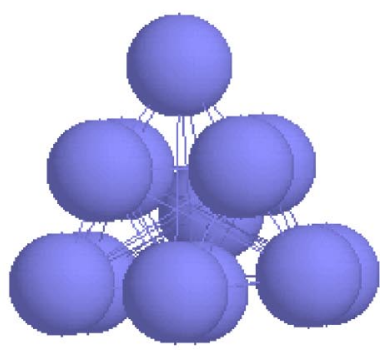

(d)

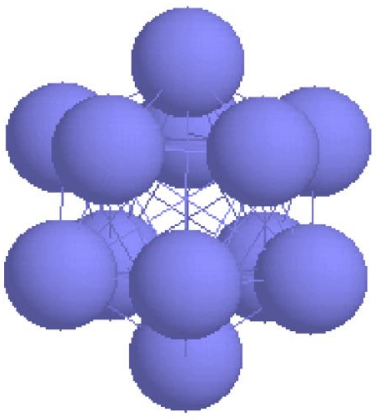

(f)
FIG. 1. The selected stable inherent structure for $X_{12}$ Lennard-Jones (LJ) cluster. Their energies (in units of the LJ well depth) are (a) -37.968, (b) -36.347 , (c) -36.209 , (d) -35.461 , (e) -34.447 , (f) -33.598 .

where $\Delta \phi_{j}$ is

$$
\Delta \phi_{j}=E_{j}^{\mathrm{TS}, \alpha}-E_{j}^{\mathrm{IS}},
$$

$E_{j}^{\mathrm{TS}, \alpha}$ is the energy of the transition state, $E_{j}^{\mathrm{IS}}$ is the energy of the inherent structure, and $\nu_{l, j}$ are the corresponding normal mode frequencies. $N$ is the number of particles in the cluster while $h_{j}$ and $h_{j}^{\alpha}$ are the order of the point group of inherent structure $j$ and transition state $\alpha$, respectively. We would like to point out that the numerical value of $\epsilon_{X X}$ and $\sigma_{X X}$ used to calculate the rate constants are $119.8 \mathrm{~K}$ and $3.405 \AA$, respectively.

\section{NUMERICAL RESULTS}

In the present section, we illustrate the general themes we introduced in Sec. I. We demonstrate that we can accomplish three basic objectives. Specifically, we show that by adding selected "impurity" atoms to bare core systems, we can (1) induce new "core structures" (2) reorder the energies

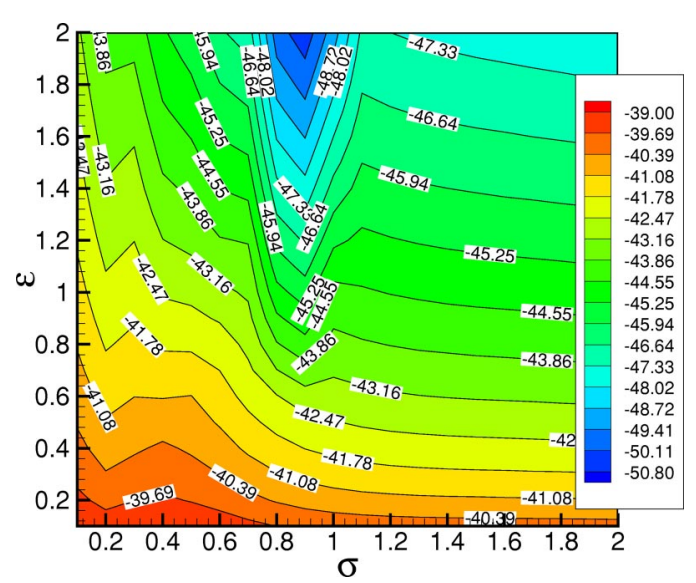

FIG. 2. $E_{\text {tot }}(\sigma, \epsilon)$ [cf. Eqs. (1), (5), and (6)] for the $X_{12} Y_{1}$ system. Note the relative lack of structure in the $(\sigma, \epsilon)$ variation of the total cluster energy.

of existing core inherent structures, and (3) stabilize selected inherent structures by controlling the activation energies that determine their isomerization kinetics.

For purposes of illustration, we examine numerical results for three, 13 atom Lennard-Jones systems involving 10, 11 , and 12 core atoms, systems well known from previous studies $^{33,34}$ to have 64,170 , and 515 energetically distinct inherent structures, respectively. These systems have been chosen because they build upon simple 10-, 11-, and 12-atom cores and because total systems have 13 atoms, a magic number for icosahedral growth in homogeneous systems.

\section{A. $X_{12} Y_{1}$}

We first consider binary clusters of the type $X_{12} Y_{1}$. The selected inherent structures and their associated energies for $X_{12}$ core system are illustrated in Fig. 1. The inherent structure labeled by (a) is the global minimum while all others are the higher lying inherent structures. Here one impurity atom $Y$ is added to the parent, 12 atom $X$ core. Using the techniques of Sec. II, we determine the lowest several inherent structures for a range of $(\sigma, \epsilon)[\mathrm{cf}$. Eqs. (5) and (6)]. As can be seen from Fig. 2, the total potential energy [Eq. (1)] of the lowest inherent structure for the $X_{12} Y_{1}$ system shows no appreciable structure as a function of the $(\sigma, \epsilon)$ parameters.

On the other hand, it can be seen in Fig. 3 that the core potential energy, defined as the potential energy of interaction for only the core $X$ atoms, of the minimum (total) energy cluster clearly breaks into extended regions, each corresponding to a well-defined core structure. We would like to point out that each region in Fig. 3 contains the same "kind" of core structure but their core energies are slightly different. We have chosen a single "average" core energy value to represent all energies in the corresponding domain for plotting convenience.

The distinct core structures, shown in Fig. 3, have been identified by examining their core energies $\left(E_{\text {core }}\right)$ and their principal moments of inertia. For each structure a triplet of values $\left(E_{\text {core }}, I_{2}, I_{3}\right)$ has been associated, where $I_{2}$ and $I_{3}$ are the moments of inertia about the principal axes 2 and 3, respectively. We have defined $I_{2}$ and $I_{3}$ in the following way: $I_{2}=I_{2}^{\prime} / I_{1}^{\prime}, I_{3}=I_{3}^{\prime} / I_{1}^{\prime}$ where $I_{1}^{\prime}, I_{2}^{\prime}$ and $I_{3}^{\prime}$ are the principal 


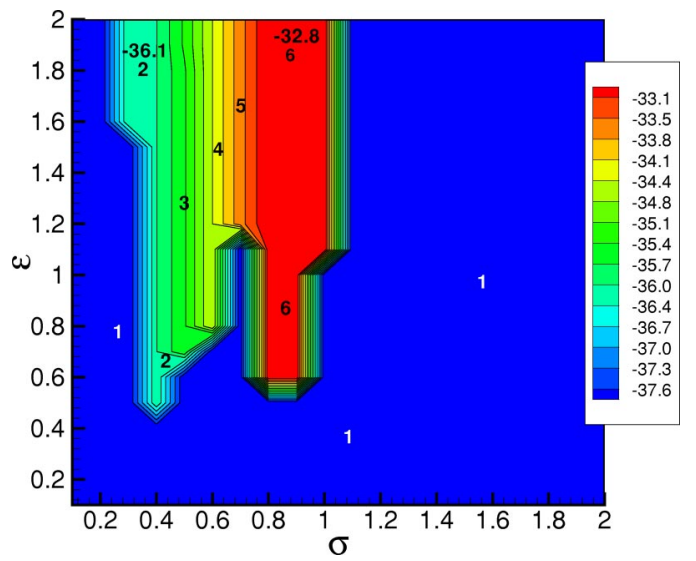

FIG. 3. $E_{\text {core }}(\sigma, \epsilon)$ for the $X_{12} Y_{1}$ system. Here the core energy is defined as that portion of the potential energy arising from only the core-core atom interactions. Unlike the total energy, the $(\sigma, \epsilon)$ variation of the core cluster energy exhibits relatively well-defined regions. The labels of each of these regions in the figure correspond to the distinct core structures shown in Fig. 4.

moments of inertia obtained by diagonalizing the inertia tensor of the system. If the triplet of values has not been sufficient to identify a core structure then we have examined the structure visually.

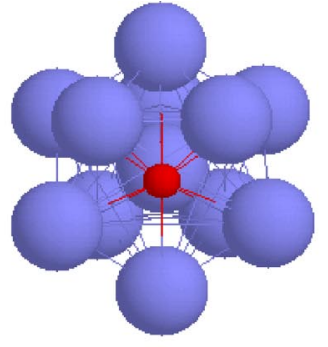

$(4.1)$

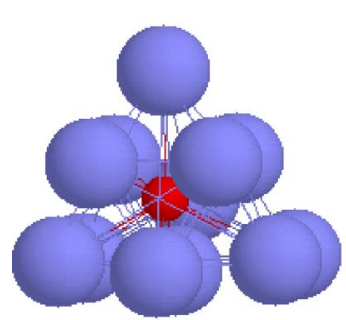

(4.3)

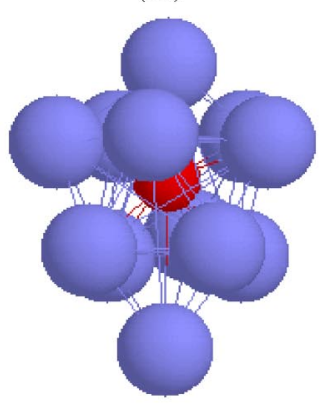

(4.5)

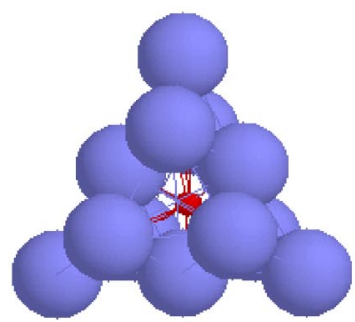

$(4.2)$

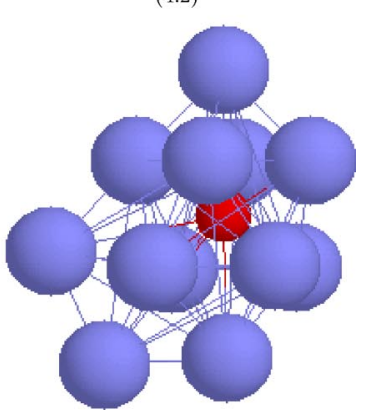

$(4.4)$

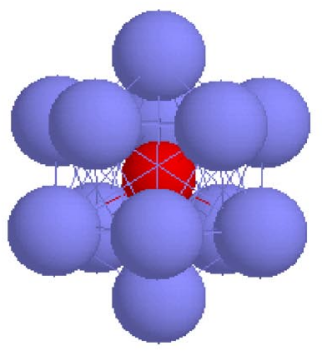

$(4.6)$
FIG. 4. Plots of $X_{12} Y_{1}$ structures for selected $(\sigma, \epsilon)$ values. The decimal number for each figure denotes the corresponding $(\sigma, \epsilon)$ domain in Fig. 3.

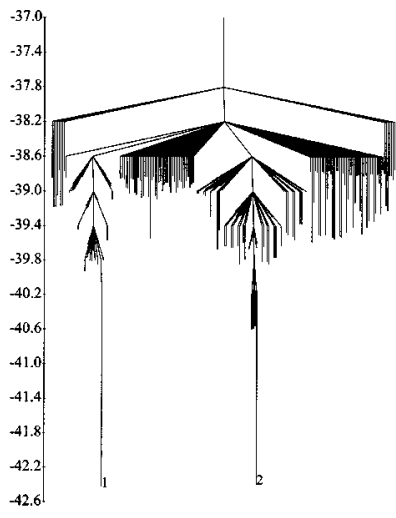

(a)

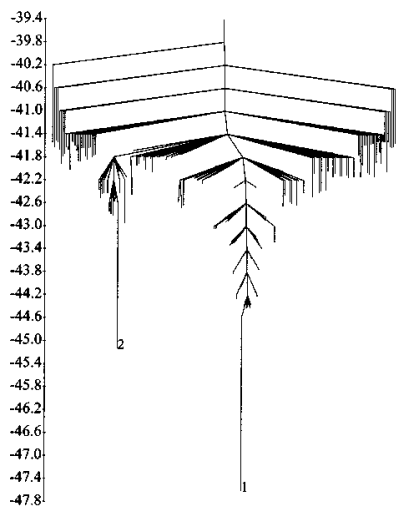

(c)

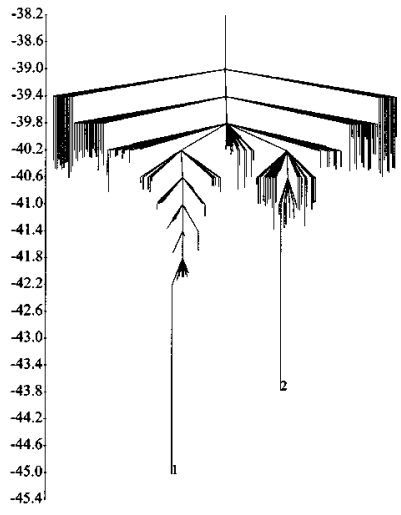

(b)

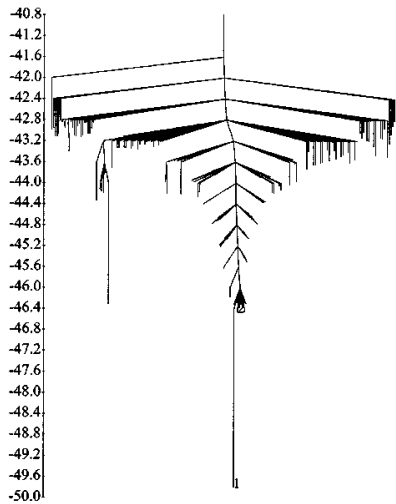

(d)
FIG. 5. Disconnectivity graph for $X_{12} Y_{1}(\sigma, \epsilon)$ values demonstrating that we can control barriers for the selected inherent structures. The energy scale is in units of $\epsilon_{X X}$. The $(\sigma, \epsilon)$ values for panels $(\mathrm{a}-\mathrm{d})$ are $(0.8,0.6),(0.8,1.0)$, $(0.8,1.5)$, and $(0.8,2.0)$, respectively. Only branches leading to the 200 lowest-energy minima are shown.

Selected cluster structures illustrating the core arrangements corresponding to various $(\sigma, \epsilon)$ values are shown in Fig. 4. It can be seen from Figs. 3 and 4 that the $X_{12} Y_{1}$ cluster exhibits core $X$-atom structures that are higher lying minima in the parent $X_{12}$ system. The core structures labeled by $(4.2),(4.3),(4.4)$, and (4.6) can be recognized as structures labeled by (c), (d), (e), and (f) in Fig. 1, respectively. This illustrates that a suitable choice of the $(\sigma, \epsilon)$ parameters can controllably reorder the energies of existing core inherent structures. The structure labeled by (4.5) shows a newly induced core geometry not present as a stable minimum in the bare cluster. These two results demonstrate that we can accomplish objectives (1) and (2) stated earlier.

Figure 5 represents the $X_{12} Y_{1}$ cluster at four points in Fig. 3 defined by the $(\sigma, \epsilon)$ co-ordinates $(0.8,0.6),(0.8,1.0)$, $(0.8,1.5)$, and $(0.8,2.0)$. Here the pairs of coordinates correspond to (a), (b), (c), and (d) of Fig. 5, respectively. In other words, we keep value of $\sigma=0.8$ fixed, while increasing the value of $\epsilon$. The number of inherent structures available to the $X_{12} Y_{1}$ cluster varies from at least 4153 in Fig. 5(a) to at least 3641 in Fig. 5(d). Since we are primarily interested in energetically low-lying inherent structures we show only the lowest 200 inherent structures. The global minimum of each system is labeled by the number 1 and contains as a recog- 
nizable component the core structure labeled by (4.6) (see Fig. 4). In Fig. 5(a) the core structure (4.6) is connected to a group of 12 inherent structures by pathways whose energies do not exceed -39.4 (in units of $\epsilon_{X X}$ ). The energies of these 12 inherent structures are very close to each other and their values are from lowest -39.810 to highest -39.614 . Their corresponding core structures are different from the core structure associated with the global minimum. This implies that barriers that connect the global minimum with the inherent structures in the group are "relevant" barriers. The relevant barriers as those that connect inherent structures that contain different core structures. By examining the disconnectivity graph on a finer energy scale we find that inherent structure 1 is connected, by the lowest isomerization barrier, to inherent structure 16 . The numerical value of the lowest isomerization barrier is $\Delta E_{1,16}=2.623 \epsilon_{X X}$. Figures 5(b) and 5(c) show that increasing the value of $\epsilon$ increases the isomerization barriers that connect inherent structure 1 with a group of 12 and 9 inherent structures, respectively. Similar to system (a), the inherent structures associated with both groups contain core structures that are different from the core structure associated with inherent structure 1. For system (b) the lowest isomerization barrier that connects inherent structure 4 with inherent structure 1 has a numerical value of $\Delta E_{1,4}$ $=2.960 \epsilon_{X X}$, while for system (c) the lowest isomerization barrier connects inherent structure 1 with inherent structure 6 and its value is $\Delta E_{1,6}=3.269 \epsilon_{X X}$. In Fig. 5(d) inherent structure 1 is connected to a group of nine inherent structures. The lowest isomerization barrier is $\Delta E_{1,3}=3.509 \epsilon_{X X}$ connecting inherent structure 1 with inherent structure 3 . The doublefunnel structure of the disconnectivity graphs is evident especially in Figs. 5(a) and 5(b) where the minima that define the two separated basins are so close in energy. The doublefunnel structure of the potential energy surface is reflected in the classical heat capacity as discussed in the companion paper. ${ }^{18}$ Below we find similar double-funnel structures for $X_{11} Y_{2}$.

We estimate the rate constants (lifetimes) for four temperatures, $5,10,100$, and $300 \mathrm{~K}$, as a function of the height of the isomerization barriers $(\epsilon)$. At the low temperatures (5 and $10 \mathrm{~K}$ ) the studied systems become extremely stable. By increasing the isomerization barrier between the global minimum and the first higher-lying inherent structure, from $\Delta E_{1,16}=2.623 \epsilon_{X X}$ [see Fig. 5(a)] to $\Delta E_{1,3}=3.509 \epsilon_{X X}$ [see Fig. 5(d)] the lifetime increases by nine and four orders of magnitude in the case of 5 and $10 \mathrm{~K}$, respectively. To be more specific, at $10 \mathrm{~K}$, the lifetime increases from the order of seconds to the order of days. This is illustrated in Fig. 14(a).

As illustrated in Figs. 5 and 14(a) the barriers that determine the isomerization kinetics are sensitive to the $(\sigma, \epsilon)$ values and can thus be at least partially controlled. Therefore, we have created selected structures that have experimentally relevant lifetimes. These results are specific demonstrations of goal (3) stated earlier.

\section{B. $X_{11} Y_{2}$}

As a second illustration, we consider mixed clusters of the type $X_{11} Y_{2}$. This system builds upon a parent, 11 atom

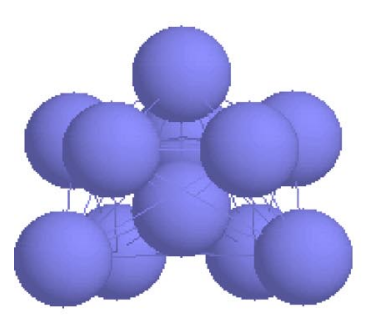

(a)

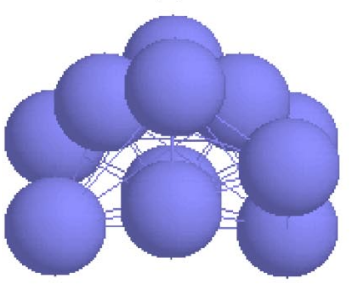

(c)

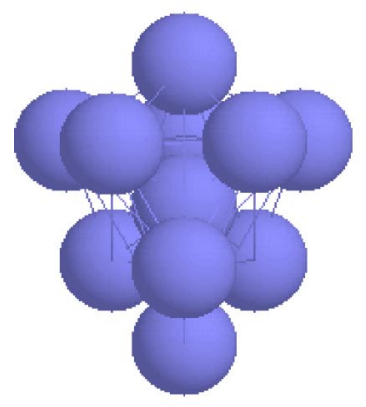

(e)

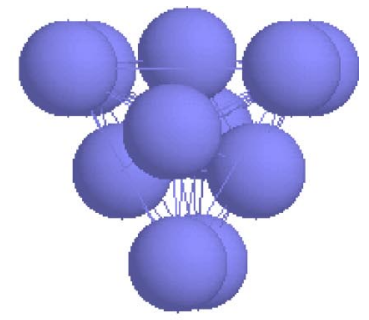

(b)

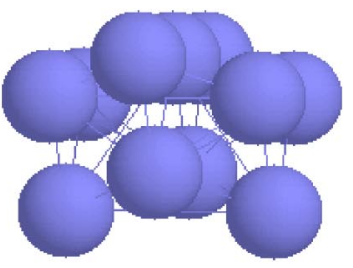

(d)

(f)

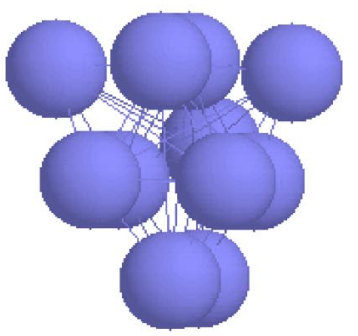

FIG. 6. The selected stable inherent structure for $X_{11} \mathrm{LJ}$ cluster. Their energies (in units of the LJ well depth) are (a) -31.766 , (b) -31.9152 , (c) -31.9146 , (d) -31.775 , (e) -31.615 , (f) -31.036 .

system known to exhibit a set of 170 , energetically distinct inherent structures. ${ }^{33}$ The selected core inherent structures and associated energies for the stable $X_{11}$ inherent structures are presented in Fig. 6. In Fig. 7, a $(\sigma, \epsilon)$ contour plot of the core-atom potential energies of the lowest total energy $X_{11} Y_{2}$ clusters, again reveals the presence of definite "core phases." As illustrated in Fig. 8, some of these regions correspond to various core structures present in the parent $X_{11}$ system while others correspond to new structures not seen in the original,

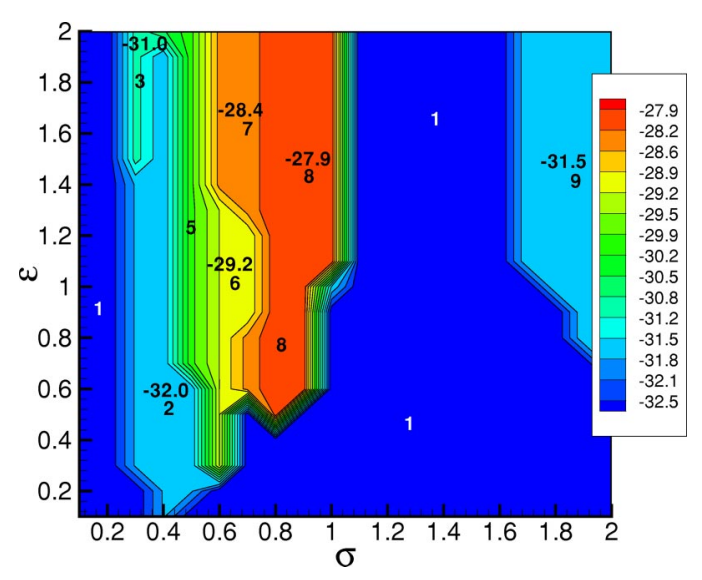

FIG. 7. $E_{\text {corc }}(\sigma, \epsilon)$ for $X_{11} Y_{2}$. Format for the plot is the same as in Fig. 3. 


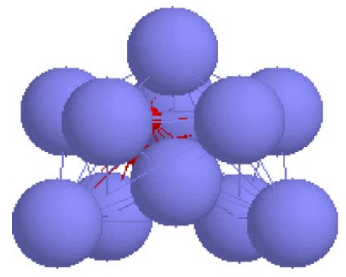

$(8.1)$

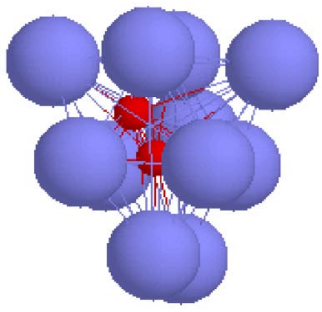

(8.4)

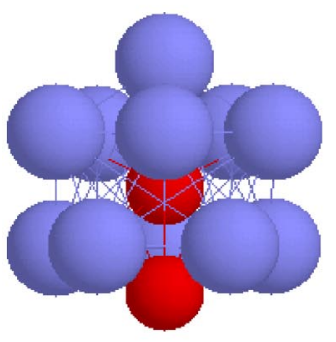

(8.8)

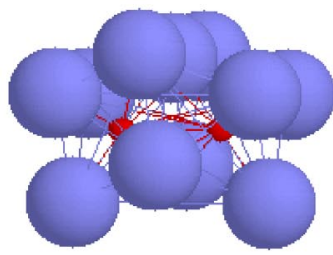

$(8.2)$

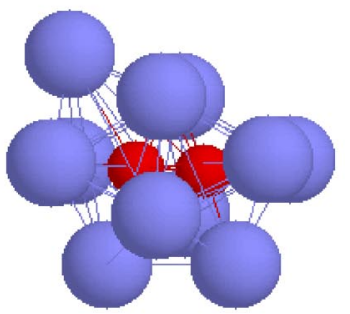

(8.6)

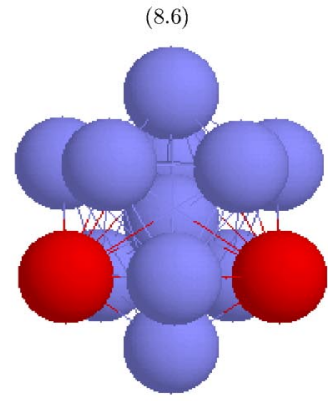

(8.9)
FIG. 8. Plots of selected $X_{11} Y_{2}$ structures for various $(\sigma, \epsilon)$ values identified in Fig. 7. The number of the structures correspond to the regions labeled in Fig. 7. The core structures for the systems labeled by (8.6) and (8.8) are not stable energy structures of the bare $X_{11}$ system.

single-component cluster. We can see from Figs. 7-9 that the impurity $Y$ atoms provide us with significant control over the relative ordering of the core energies of the parent $X_{11}$ system. Moreover, since we can manipulate the isomerization barriers in the $X_{11} Y_{2}$ systems, we can at least partially stabilize clusters that exhibit selected core structures with respect to isomerization. This is illustrated in Fig. 9.

Figures 9(a)-9(d) represent the $X_{11} Y_{2}$ cluster at four points in Fig. 7 with $X_{11} Y_{2}(\sigma, \epsilon)$ coordinates $(0.8,0.5)$, $(0.8,1.0),(0.8,1.5)$, and $(0.8,2.0)$, respectively. The number of inherent structures available to the $X_{11} Y_{2}$ cluster in all four cases is more than 6000 . We show only the lowest 200 inherent structures. The global minimum of each system is labeled by the number 1 and contains as a recognizable component the core structure (8.8) shown in Fig. 8. In Fig. 9(a) the global minimum, the core structure labeled by (8.8) in Fig. 8, is linked to inherent structure 5. Its core structure is different from the one associated with the global minimum. The isomerization barrier between them is $\Delta E_{1,5}$ $=1.015 \epsilon_{X X}$. In Fig. 9(b) inherent structure 1 is connected to inherent structures 3 and 4 whose energies are almost degenerate. Both inherent structures 3 and 4 contain core structures that are different from each other and from the one associated with inherent structure 1 . The isomerization barriers between inherent structures 1 and 3 and 1 and 4 are $\Delta E_{1,3}$ $=2.025 \epsilon_{X X}$ and $\Delta E_{1,4}=2.011 \epsilon_{X X}$, respectively. Figures $9(\mathrm{c})$

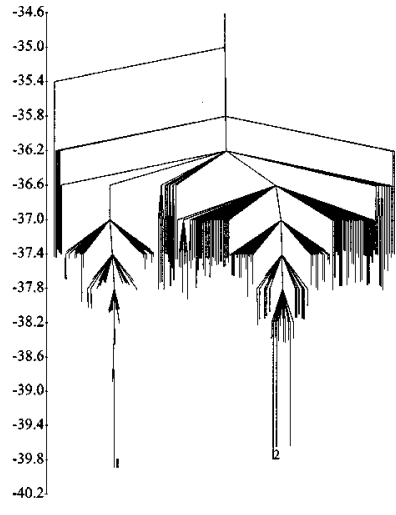

(a)

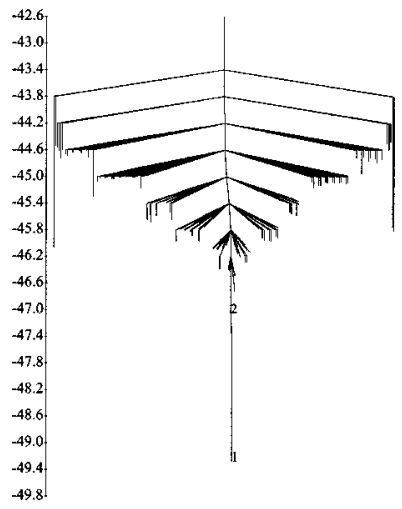

(c)

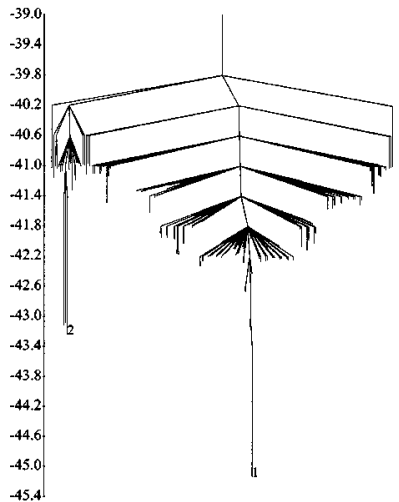

(b)

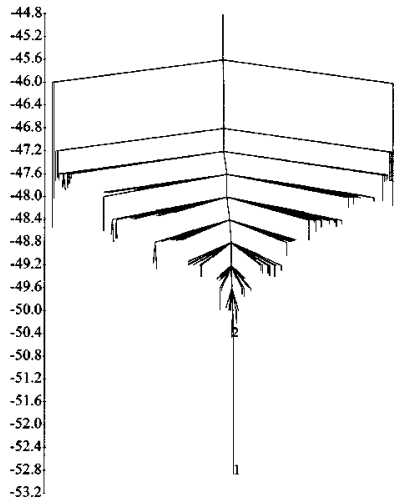

(d)
FIG. 9. Disconnectivity graph for $X_{11} Y_{2}(\sigma, \epsilon)$ values demonstrating that we can control barriers for the selected inherent structures. The energy scale is in units of $\epsilon_{X X}$. The $(\sigma, \epsilon)$ values for panels (a)-(d) are $(0.8,0.5),(0.8,1.0)$, $(0.8,1.5)$, and $(0.8,2.0)$, respectively. Only branches leading to the 200 lowest-energy minima are shown.

and 9(d) show that further increasing of the value of $\epsilon$ increases isomerization barriers that link inherent structure 1 with inherent structure 2 . Numerically, these barriers are $\Delta E_{1,2}=2.265 \epsilon_{X X}$ and $\Delta E_{1,2}=2.463 \epsilon_{X X}$, respectively. Estimated lifetimes are shown in Fig. 14(b) as a function of $\epsilon$. It can be seen that the lifetimes increase by fourteen and seven orders of magnitude at 5 and $10 \mathrm{~K}$, respectively.

\section{C. $X_{10} Y_{3}$}

As a third illustration, we consider mixed clusters of the type $X_{10} Y_{3}$. This system builds upon a parent, 10 atom, system known to exhibit a set of 64, energetically distinct inherent structures. ${ }^{33}$ The selected core inherent structures and associated energies for the stable $X_{10}$ inherent structures are presented in Fig. 10. A $(\sigma, \epsilon)$ contour plot of the core-atom potential energies of the lowest total energy $X_{10} Y_{3}$ clusters is shown in Fig. 11. In other words, Fig. 11 (and in a similar manner Figs. 3 and 7) shows clearly how one can choose a pair of coordinates $(\sigma, \epsilon)$ in the $\sigma \epsilon$ plane so that a target structure can be obtained. For example, in order to obtain the core structure shown in Fig. 12.2 [or in Fig. 10(c)] one needs to fix the value of $\sigma=0.3$ and move up in $\epsilon \in[0.4,2.0]$. As illustrated in Fig. 12 some of the domains in Fig. 11 correspond to the core structures present in the parent 

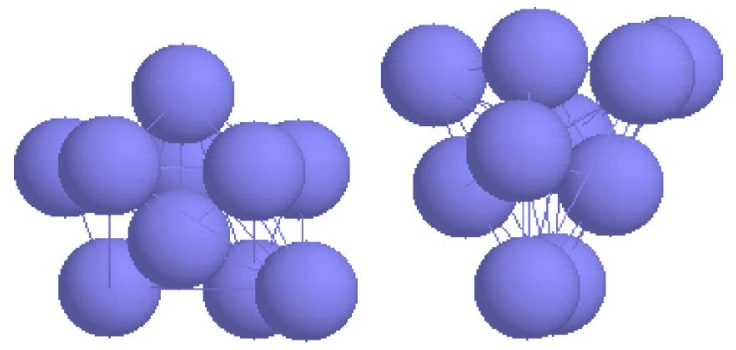

(a)

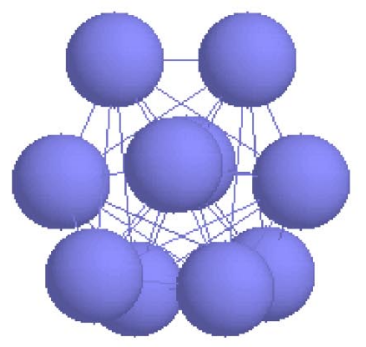

(c)

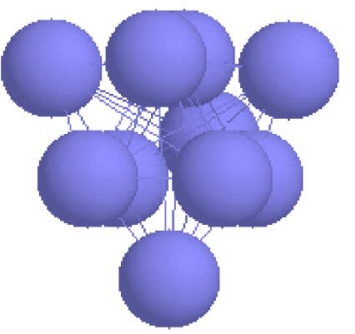

(e) (b)

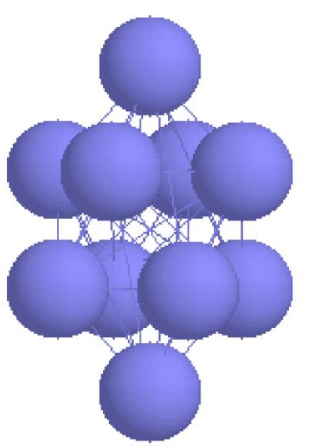

(d)

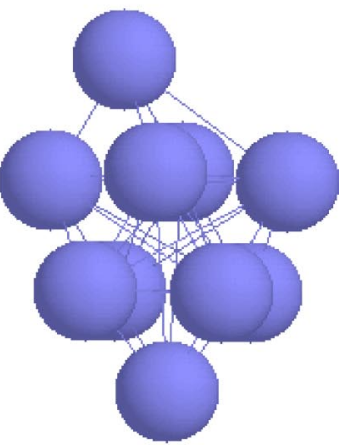

(f)

FIG. 10. The selected stable inherent structure for $X_{10} \mathrm{LJ}$ cluster. Their energies (in units of the LJ well depth) are (a) -28.422 , (b) -27.556 , (c) -27.214 , (d) -26.772 , (e) -26.698 , (f) -26.695 .

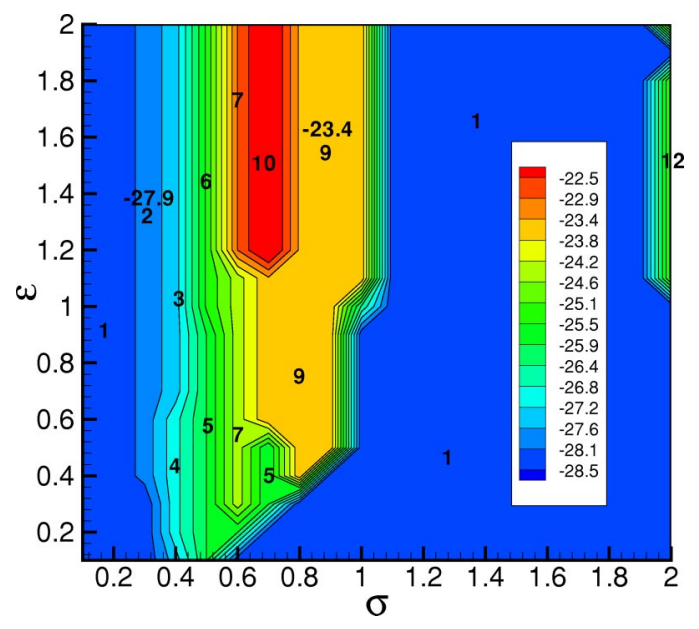

FIG. 11. $E_{\text {core }}(\sigma, \epsilon)$ for $X_{10} Y_{3}$. Format for the plot is the same as in Figs. 3 and 7.

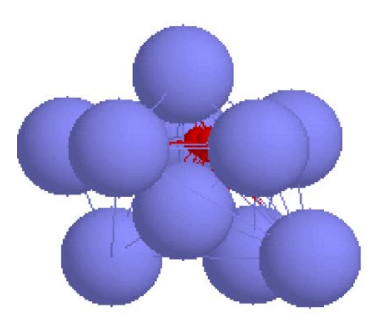

(12.1)

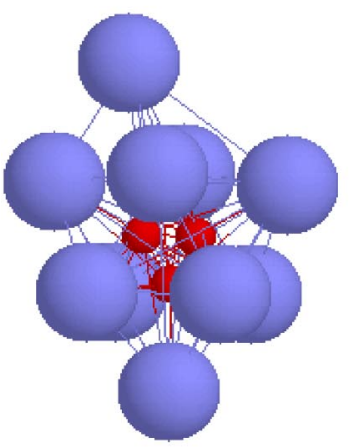

(12.3)

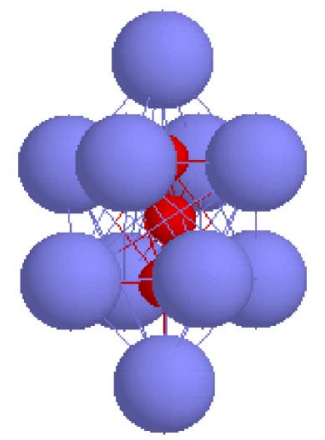

$(12.5)$

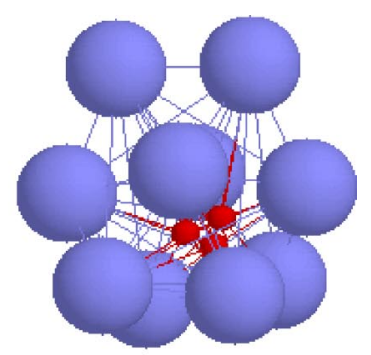

(12.2)

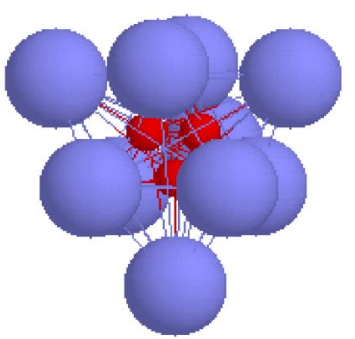

(12.4)

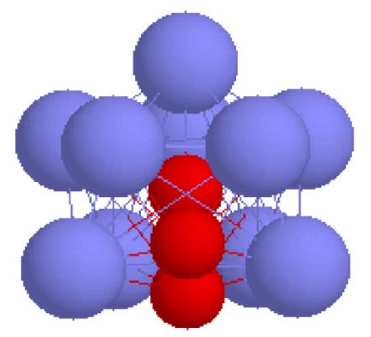

(12.9)
FIG. 12. Plots of selected $X_{10} Y_{3}$ structures for various $(\sigma, \epsilon)$ values identified in Fig. 11. The number of the structures correspond to the regions labeled in Fig. 11. The core structure for the system labeled by (12.9) is not a stable energy structure of the bare $X_{10}$ system.

system $X_{10}$ while others correspond to new structures not seen in original, single-component cluster. Specifically, the domains $1,2,3,4$, and 5 correspond to the core structures labeled by (a), (c), (f), (e), and (d) in Fig. 10, respectively. Therefore, the impurity atoms $Y$ provide significant control over relative ordering of the core energies of the parent $X_{10}$ system. Figure 13 illustrates that, by choosing the appropriate set of $(\sigma, \epsilon)$ values we can manipulate the isomerization barriers in the selected systems.

Figures 13(a)-13(d) represent the $X_{10} Y_{3}$ cluster at four points in Fig. 11 with $X_{10} Y_{3}(\sigma, \epsilon)$ coordinates $(0.8,0.5)$, $(0.8,1.0),(0.8,1.5)$, and $(0.8,2.0)$, respectively. The number of inherent structures available to the $X_{10} Y_{3}$ cluster in all four cases is more than 6000 . We show only the lowest 200 inherent structures. The global minimum of each system is labeled by the number 1 and contains as a recognizable component the core structure (12.9) shown in Fig. 12. In Fig. 9(a) inherent structure 1 is linked to inherent structure 6 with the isomerization barrier which value is $\Delta E_{1,6}=0.779 \epsilon_{X X}$. The 


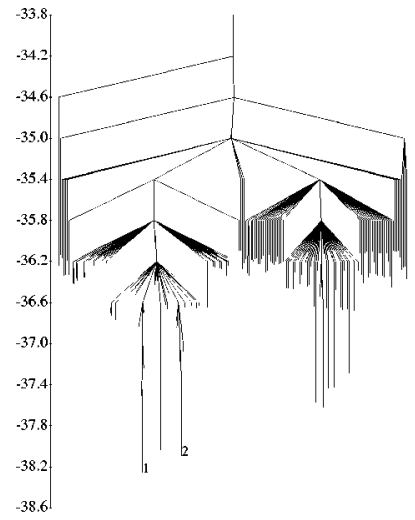

(a)

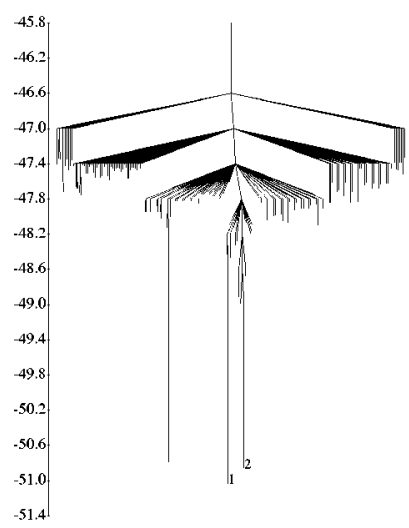

(c)

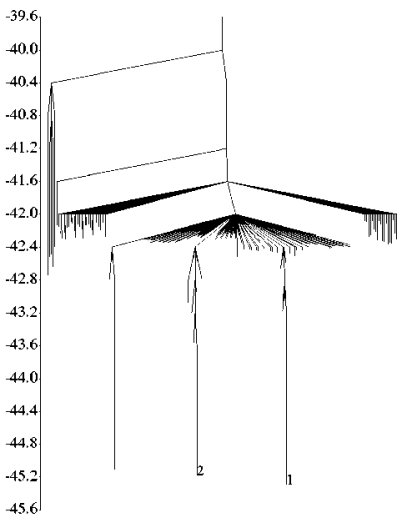

(b)

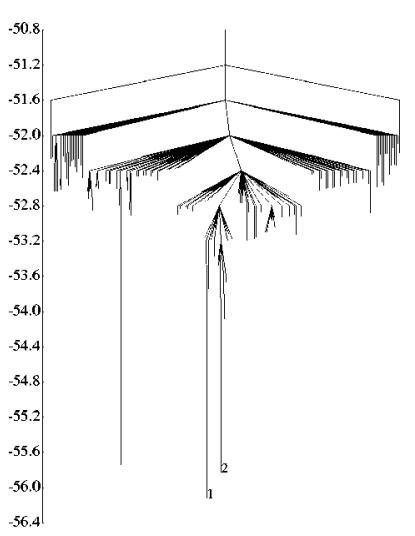

(d)

FIG. 13. Disconnectivity graph for $X_{10} Y_{3}(\sigma, \epsilon)$ values demonstrating that we can control barriers for the selected inherent structures. The energy scale is in units of $\epsilon_{X X}$. The $(\sigma, \epsilon)$ values for panels (a)-(d) are $(0.8,0.5),(0.8,1.0)$, $(0.8,1.5)$, and $(0.8,2.0)$, respectively. Only branches leading to the 200 lowest-energy minima are shown.

core structure corresponding to inherent structure 6 is different from the core structure corresponding to the global minimum. In Fig. 13(b) inherent structure 1 is connected to inherent structures 7 and 9 whose energy values are $-43.177 \epsilon_{X X}$ and $-43.034 \epsilon_{X X}$, respectively. Both inherent structures 7 and 9 contain core structures that are different from each other and from the one associated with inherent structure 1. The isomerization barriers between inherent structures 1 and 7 and 1 and 9 are $\Delta E_{1,7}=2.138 \epsilon_{X X}$ and $\Delta E_{1,9}=2.252 \epsilon_{X X}$, respectively. Figures $9(\mathrm{c})$ and $9(\mathrm{~d})$ show that increasing of the value of $\epsilon$ increases isomerization barriers that link inherent structure 1 with inherent structures 9 and 12 , respectively. Numerically, these barriers are $\Delta E_{1,9}$ $=2.630 \epsilon_{X X}$ and $\Delta E_{1,12}=2.883 \epsilon_{X X}$, respectively. It can be seen from Fig. 14(c) that by increasing the height of isomerization barriers the lifetime can increase by 21 and 10 orders of magnitude at 5 and $10 \mathrm{~K}$, respectively. These results are specific demonstration of goal (3) stated earlier.

\section{CONCLUSIONS}

As stated at the outset, the general theme of the present work is to explore the extent to which one can induce controllable structural modifications in clusters. One reason for
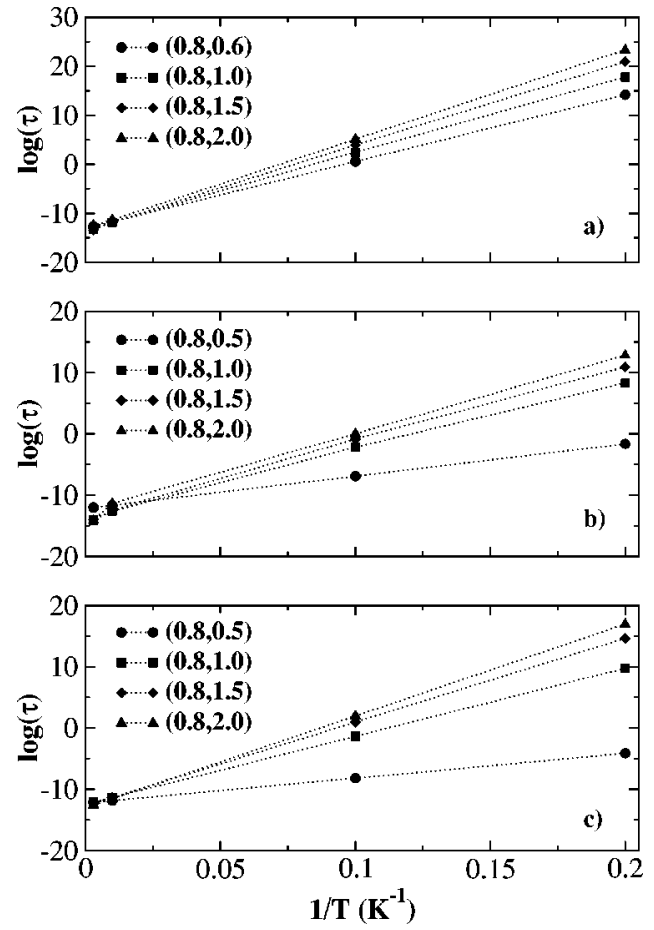

FIG. 14. Temperature dependence of the logarithm (base 10) of lifetime $\tau$ (units seconds), for the selected inherent structures, as a function of different $\epsilon$ values: (a) $X_{12} Y_{1}(\sigma, \epsilon)$, (b) $X_{11} Y_{2}(\sigma, \epsilon)$, (c) $X_{10} Y_{3}(\sigma, \epsilon)$.

pursuing such a development is the possibility that such modifications might be a general technique for producing materials that have "interesting" properties (electronic; magnetic, optical, thermal, etc.). Depending on the application, one could envision such clusters being of use either directly, or, if they could be made sufficiently robust, as precursors in subsequent assembly of yet more complex materials.

Both current and previous work ${ }^{11}$ indicate that such controllable modifications are possible in model Lennard-Jones systems. In particular, we have demonstrated that by introducing impurity atoms of varying size and interaction energies, we can produce core-atom conformers that correspond to a variety of nonminimum energy homogeneous isomers. We have also shown that it is also possible to use such impurities to generate core-atom structures that posses no (stable) homogeneous analogs. We have now demonstrated such capabilities for both the simple $X_{5} Y_{2}$ and $X_{7} Y_{3}$ systems $^{11}$ and for the larger, more complex $X_{12} Y_{1}, X_{11} Y_{2}$, and $X_{10} Y_{3}$ binary clusters.

Finally, in the present work we have investigated the issue of the stability of our modified clusters. Based on simple transition-state estimates of the rates of isomerization of the various clusters, we have demonstrated that it is possible both to induce and to stabilize a variety of structural modifications. In the companion ${ }^{18}$ paper we investigate how the structures of the underlying potential energy surfaces explored in this work are reflected in the thermodynamic properties of the systems.

\section{ACKNOWLEDGMENTS}

The authors acknowledge support from the National Science Foundation through Award Nos. CHE-0095053 and 
CHE-0131114. They wish to thank Professor David Wales for providing a copy of an executable file of his program for determination of a point group symmetry of the clusters. One of us (D.S.) is grateful to Cristian Diaconu for helpful discussions. They would also like to thank Dr. M. Miller for helpful discussions and for his gracious assistance with respect to the preparation of the disconnectivity graphs in the present paper. Finally, the authors would like to thank the Center for Advanced Scientific Computing and Visualization at Brown University for their assistance.

${ }^{1}$ D. J. Wales, J. P. K. Doye, M. A. Miller, P. N. Mortenson, and T. R. Walsh, Adv. Chem. Phys. 115, 1 (2000).

${ }^{2}$ J. P. Neirotti, F. Calvo, D. L. Freeman, and J. D. Doll, J. Chem. Phys. 112, 10340 (2000).

${ }^{3}$ F. Calvo, J. P. Neirotti, D. L. Freeman, and J. D. Doll, J. Chem. Phys. 112, 10350 (2000).

${ }^{4}$ L. J. Munro, A. Tharrington, and K. D. Jordan, Comput. Phys. Commun. 145, 1 (2002)

${ }^{5}$ A. N. Tharrington and K. D. Jordan, J. Phys. Chem. A 107, 7380 (2003).

${ }^{6}$ S. Sun, C. B. Murray, D. Weller, L. Folks, and A. Moser, Science 287, 1989 (2000).

${ }^{7}$ K. K. Lehmann and G. Scoles, Science 279, 2065 (1998).

${ }^{8}$ K. Nauta and R. E. Miller, Science 283, 1895 (1999).

${ }^{9}$ K. Nauta and R. E. Miller, Phys. Rev. Lett. 82, 4480 (1999).

${ }^{10}$ K. Nauta, D. T. Moore and R. E. Miller, Faraday Discuss. 113, 261 (1999).

${ }^{11}$ D. Sabo, J. D. Doll, and D. L. Freeman, J. Chem. Phys. 118, 7321 (2003).

${ }^{12}$ P. Nigra, D. L. Freeman, D. Sabo, and J. D. Doll, J. Chem. Phys. (to be published).

${ }^{13}$ I. Bytheway and D. L. Kepert, J. Math. Chem. 9, 161 (1992).

${ }^{14}$ F. Calvo and E. Yurtsever, http://arXiv.org/abs/physics/0403048
${ }^{15}$ D. J. Wales, Energy Landscapes with Applications to Clusters, Biomolecules and Glasses (Cambridge University Press, New York, 2003).

${ }^{16} \mathrm{~J}$. Jellinek and E. B. Krissinel, in Theory of Atomic and Molecular Clusters, edited by J. Jellinek (Springer, Berlin, 1999).

${ }^{17}$ J. P. K. Doye, D. J. Wales, and M. A. Miller, J. Chem. Phys. 109, 8143 (1998).

${ }^{18}$ D. Sabo, C. Predescu, J. D. Doll, and D. L. Freeman, J. Chem. Phys. 121, 856 (2004).

${ }^{19}$ J. O. Hirschfelder, C. F. Curtiss, and R. B. Bird, Molecular Theory of Gases and Liquids (Wiley, New York, 1954), p. 168.

${ }^{20}$ W. H. Press, S. A. Teukolsky, W. T. Vetterling, and B. P. Flannery, Numerical Recipes, 2nd ed. (Cambridge University Press, Cambridge, 1992).

${ }^{21}$ J. P. K. Doye, M. A. Miller, and D. J. Wales, J. Chem. Phys. 110, 6896 (1999).

${ }^{22}$ C. J. Tsai and K. D. Jordan, J. Phys. Chem. 97, 11227 (1993).

${ }^{23}$ C. J. Cerjan and W. H. Miller, J. Chem. Phys. 75, 2800 (1981).

${ }^{24}$ J. Simons, P. Jorgensen, H. Taylor, and J. Ozment, J. Phys. Chem. 87, 2745 (1983).

${ }^{25}$ A. Banerjee, N. Adams, J. Simons, and R. Shepard, J. Phys. Chem. 89, 52 (1985).

${ }^{26}$ J. Nichlos, H. Taylor, P. Schmidt, and J. Simons, J. Chem. Phys. 92, 340 (1990).

${ }^{27}$ D. J. Wales, J. Chem. Phys. 101, 3750 (1994).

${ }^{28}$ O. M. Becker and M. Karplus, J. Chem. Phys. 106, 1495 (1997).

${ }^{29}$ M. A. Miller, J. P. K. Doye, and D. J. Wales, J. Chem. Phys. 110, 328 (1999).

${ }^{30}$ R. E. Kunz and R. S. Berry, J. Chem. Phys. 103, 1904 (1995).

${ }^{31}$ J. P. Rose and R. S. Berry, J. Chem. Phys. 96, 517 (1992).

${ }^{32}$ F. Calvo, F. Spiegelmann, and D. J. Wales, J. Chem. Phys. 118, 8754 (2003).

${ }^{33}$ D. B. Faken, Ph.D. thesis, Brown University, 2001.

${ }^{34}$ J. P. K. Doye and D. J. Wales, J. Chem. Phys. 116, 3777 (2002). 
The Journal of Chemical Physics is copyrighted by the American Institute of Physics (AIP). Redistribution of journal material is subject to the AIP online journal license and/or AIP copyright. For more information, see http:/ojps.aip.org/jcpo/jcpcr/jsp Copyright of Journal of Chemical Physics is the property of American Institute of Physics and its content may not be copied or emailed to multiple sites or posted to a listserv without the copyright holder's express written permission. However, users may print, download, or email articles for individual use. 
The Journal of Chemical Physics is copyrighted by the American Institute of Physics (AIP). Redistribution of journal material is subject to the AIP online journal license and/or AIP copyright. For more information, see http://ojps.aip.org/jcpo/jcper/jsp 